\title{
Impact of COVID-19 restrictions on alcohol consumption behaviours
}

\author{
Emily O. C. Palmer, William Trender, Robin J. Tyacke, Adam Hampshire and Anne Lingford-Hughes
}

\section{Background}

We aimed to evaluate how coronavirus (COVID-19) restrictions had altered individual's drinking behaviours, including consumption, hangover experiences, and motivations to drink, and changing levels of depression and anxiety.

\section{Method}

We conducted an online cross-sectional self-report survey. Whole group analysis compared pre- versus post-COVID restrictions. A correlation coefficient matrix evaluated the associations between all outcome scores. Self-report data was compared with Alcohol Use Disorders Identification Test (AUDIT) scores from the 2014 Adult Psychiatric Morbidity Survey. Multiple linear modelling (MLM) was calculated to identify factors associated with increasing AUDIT scores and post-restriction AUDIT scores.

\section{Results}

In total, 346 individuals completed the survey, of which 336 reported drinking and were therefore analysed. After COVID-19 restrictions $23.2 \%$ of respondents reported an increased AUDIT score, and $60.1 \%$ a decreased score. AUDIT score change was positively correlated with change in depression $(P<0.01$, $r=0.15)$, anxiety $(P<0.01, r=0.15)$ and drinking to cope scores
$(P<0.0001, r=0.35)$. MLM revealed that higher AUDIT scores were associated with age, mental illness, lack of a garden, selfemployed or furloughed individuals, a confirmed COVID-19 diagnosis and smoking status.

\section{Conclusions}

COVID-19 restrictions decreased alcohol consumption for the majority of individuals in this study. However, a small proportion increased their consumption; this related to drinking to cope and increased depression and anxiety.

\section{Keywords}

Alcohol disorders; cognitive neuroscience; psychosocial interventions; drugs of dependence disorders; epidemiology.

\section{Copyright and usage}

(c) The Author(s), 2021. Published by Cambridge University Press on behalf of the Royal College of Psychiatrists. This is an Open Access article, distributed under the terms of the Creative Commons Attribution licence (http://creativecommons.org/ licenses/by/4.0/), which permits unrestricted re-use, distribution, and reproduction in any medium, provided the original work is properly cited.

\section{Background}

Coronavirus (COVID-19) has changed social behaviours on a global scale. A recent report from the Office of National Statistics reported that $37.4 \%$ of adults in Great Britain said the COVID-19 pandemic has affected their well-being. ${ }^{1}$ Indeed, a recent longitudinal study found that mental health problems assessed using the General Health Questionnaire-12 (GHQ-12) have increased substantially (from $23.4 \%$ in $2017-2019$ to $37.1 \%$ in April 2020). ${ }^{2}$ In addition to growing mental health concerns, others anticipated that the wider implications of the COVID-19 pandemic will lead to an increase in alcohol and substance use disorders. ${ }^{3,4}$ For instance, recent data from Belgium suggests that COVID-19 lockdown measures resulted in increased consumption of both alcohol and tobacco ${ }^{5}$ and data from Public Health England and analysed by the Royal College of Psychiatry found that over 8.4 million people are now drinking amounts that put them at higher risk of ill health (assessed using a survey enquiring about units consumed in a typical week) compared with 4.8 million in February $2020 .^{6}$ Similarly, a recent commentary noted that across a dozen studies $20-40 \%$ of surveyed participants reported drinking more. ${ }^{7}$

Such increased consumption may also adversely have an impact on vulnerability to COVID-19 as there is evidence that alcohol may disrupt the immune response. ${ }^{8}$ In addition, increased consumption is likely to translate to increased alcohol-specific deaths. Indeed, the Office of National Statistics reported that 2020 had the highest annual total of alcohol-specific deaths since the start of the dataseries in 2001 (accessed June 2021). ${ }^{9}$ Indeed, deaths increased 19.6\% compared with 2019 and have been increasing with every successive quarter. Further investigation is therefore required to understand the interplay between COVID-19 and alcohol consumption.
It has previously been shown that drinking motivations can be altered in response to the context of social facilitation. ${ }^{10}$ Drinking motivations have been linked to risk of developing alcohol use disorder. ${ }^{11}$ COVID restrictions have not affected all individuals equally, ${ }^{12}$ therefore certain characteristics may be related to increased alcohol consumption among some individuals. It is therefore critical to evaluate the relationship between drinking motivations, individual characteristics, mental health and increased alcohol consumption in response to the COVID-19 pandemic.

\section{Aims}

We developed a self-report survey with the aim of understanding how pandemic-related restrictions altered alcohol consumption, motivations to drink as well as depression and anxiety. We hypothesised that COVID-19 restrictions would increase overall alcohol consumption, and that this would be associated with an increase in the motivation to drink to cope, and anxiety and depression scores. We predicted that because of the removal of social drinking occasions there would be a reduction in binge drinking (six or more drinks on one drinking occasion) occurrences, but that individuals would be drinking more regularly.

\section{Method}

\section{Participants}

Participants were recruited between 4 June and 5 August 2020 via social media platforms (Instagram, Facebook and Twitter). Participants could complete the survey if they had internet access and were English speaking. Participants younger than 16 years old 
were shown a message indicating some parts of the survey may be irrelevant to them but they were allowed to participate (for example financial security questions). However, no respondents were 16 years old or younger. Prior to completing the survey participants were required to provide informed consent (by ticking boxes online) to participate in the survey. All data was collected anonymously.

\section{Procedures}

The survey was launched and run from the hangover.cognitron.co. uk website. Participants completed a single survey divided into three sections: background, pre-COVID-19 and post-COVID-19. The background section collected demographic information, employment status, country and details of current implemented COVID19 restrictions and financial security. Participants were instructed to answer questions regarding their emotions and behaviours before the implementation of COVID-19 restrictions (based on their recall) followed by the same questions after the implementation of COVID-19 restrictions (current emotions and behaviours). The participant determined which COVID-19 restrictions they were referring to rather than specify a particular date or action because of the multiplicity of different approaches experienced by individuals in various countries. Participants were then asked, 'In this section of the survey please respond with what is correct for you AFTER you started following the COVID-19 related restrictions you have previously described' (henceforth referred to as 'COVID-19 restrictions'). The survey was designed to take about 20-25 min to complete (on average it took $13 \mathrm{~min}$ ). Participants were able to stop taking part at any stage, only completed survey data was collected.

Approval was obtained from Imperial College Research Ethics Committee, reference: 20IC5961. The study was conducted in accordance with the recommendations for research on human participants adopted by the 18th World Medical Assembly, Helsinki 1964 and later revisions.

\section{Measures}

The Alcohol Use Disorders Identification Test (AUDIT) was used to evaluate alcohol consumption, drinking behaviours and alcoholrelated problems. ${ }^{13,14}$ The self-report nine-item Patient Health Questionnaire (PHQ-9) and seven item Generalized Anxiety Disorder (GAD-7) assessed depression and anxiety, respectively. ${ }^{15,16}$ For both measures scores of 5, 10, 15 and 20 represent mild, moderate, moderately severe and severe thresholds. ${ }^{15-17}$

The 20-item Drinking Motives Questionnaire, Revised (DMQ$\mathrm{R})$ assessed the participant's motives to consume alcohol using a 1-5 scale from 'almost never/never' to 'almost always/always'. ${ }^{18}$ This questionnaire has four subscales: social, coping, conformity and enhancement. To determine sensitivity to hangovers, participants were asked 'Do you experience hangovers?' requiring a simple yes/no response. We developed a Hangover Questionnaire to evaluate drinking behaviours that induce a hangover (Supplementary Material 1 available at https://doi.org/10.1192/bjo.2021.986).

Participants were asked to rate their concern about COVID-19 over the past 2 weeks compared with before the COVID-19 restrictions (Supplementary Material 2). This gave a total COVID concern score for which the maximum was 48 , and 24 indicated no change from normal.

\section{Analysis}

All analyses were completed using SPSS (v25, 2017) and Prism (v9, 2020). Prior to analysis all variables were tested for normality. Several of the data-sets were not normally distributed and therefore non-parametric tests were used for the analysis. The outcome score 'change' refers to the difference in self-report scores pre- versus post-COVID-19 restrictions. The Wilcoxon signed-rank test was used to compare whole group pre-restriction data with post-restriction data. Spearman rank correlation coefficient matrix was used to evaluate the strength of associations between all outcome scores with the significance cut-off of $P<0.05$. Given the possible effects of recall bias the AUDIT scores from our participants were compared with previous population results from the publicly available data from the Adult Psychiatric Morbidity Survey (APMS). ${ }^{19}$

Multiple linear modelling (MLM) tested how cohort demographics and pandemic specific factors (such as age, gender, concern about finances because of the pandemic) were associated with change in AUDIT scores and post-restriction AUDIT scores. We looked at both AUDIT change and post-restriction AUDIT scores to account for a potential effect of recall bias. To help identify if the direction of change in drinking consumptions (increase versus decrease) was influenced by the pre-existing drinking behaviours an ANCOVA with post hoc multiple comparisons was used to mitigate the effect of regression to the mean (RTM). All figures are given as mean (s.d.) unless otherwise specified.

\section{Results}

In total, 346 participants responded to the survey, of which 10 were teetotal so their data was not analysed further leaving data from 336 participants. All demographic data and COVID-19-related participant characteristics are shown in Table 1 . The majority of respondents were female $(64.3 \%)$, between age of 21 and $40(85.8 \%)$, White $(90.5 \%)$, residence in the UK $(75.6 \%)$ in a relationship $(41.7 \%)$ and achieved an undergraduate or postgraduate degree (84.9\%).

As a group, AUDIT scores were significantly lower after COVID-19 restrictions and fell below the threshold considered 'harmful or hazardous' (Table 2). Interestingly, COVID-19 restrictions increased scores for anxiety and depression to above the respective 'mild' diagnosis thresholds (GAD-7 > 5, PHQ-9 > 5). Almost a quarter $(23.2 \%)$ of respondents reported an increase in AUDIT score pre- to post-COVID-19, but $60.1 \%$ reported a decreased score.

Of the individual factors of the AUDIT, quantity of alcohol consumed and binge drinking were significantly decreased, but the frequency of consumption remained unchanged pre- versus post-COVID restrictions (Table 2). Further, respondents reported that hangover frequency and the number of drinks required to induce a hangover was significantly reduced. As shown in Table 2, all the drinking motives were significantly decreased except 'Coping' which remained the same.

The AUDIT scores from our participants were compared with previous national population results (with non-drinkers removed) from the APMS (Supplementary Material 3). ${ }^{20}$ The pre-restriction self-report AUDIT scores suggest a significantly higher proportion of hazardous and harmful and dependant drinkers $(38 \%, 10 \%$ and $5 \%$, respectively) when compared with APMS results $(22 \%, 2 \%$ and $2 \%$, respectively). However, when the APMS data is compared with the proportion of hazardous and harmful and dependant drinkers reported in the post-restriction AUDIT scores (21\%, 4\% and $6 \%$, respectively) they are in close agreement.

The strength of associations between drinking behaviour, motivations to drink, and anxiety and depression scores were evaluated using Spearman rank correlation analysis Fig. 1( $a$ and b). Figure 1(a) illustrates significant positive correlations between the change in AUDIT score and the change in depression $(r=0.15, P<0.01)$, anxiety $(r=0.15, P<0.01)$ and drinking to cope scores $(r=0.35$, 


\section{Table 1 Participants characteristics}

\section{Characteristics}

\section{Age, years}

18-20

21-25

26-30

$31-40$

$41-50$

51-60

$>60$

Gender

Male

Female

Other

Prefer not to say

Ethnicity

White

Other ethnic background

Marital status

Married

In a relationship

Single

Other

Highest educational level

school

college

Undergraduate degree

Master's degree (Or equivalent)

PhD

Other

Alcohol dependence (self-report not from AUDIT)

Currently dependent

Previously dependent

No history of dependence

Other

Illicit drug dependence (self-report)

Currently dependent

Previously dependent

No history of dependence

Other

Smoking (tobacco) status

Smoker

Non-smoker

Country of residence

UK

USA

Other

Residential area

City

Suburban

Rural

Residence type

Flat or apartment

House without garden

House with garden

Other

Number of people in residence

1

2

3

4 or more

\section{$n$}

7

85

80

77

38

24

24

116

216

3

1

304

32

97

140

96

2

14

33

146

99

40

4

11

11

312

2

5

312

65

271

254

46

36

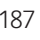

92

57

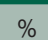

2.1

30.1

28.4

27.3

13.5

8.5

8.9

34.5

64.3

0.9

0.3

90.5

9.5

28.9

41.7

28.6

0.6

4.2

9.8

43.5

29.5

11.9

1.2

3.3

3.3

92.9

0.6

1.5

4.5

92.9

1.2

19.3

80.7

75.6

13.7

10.7

55.7

27.4

17.0

37.2

4.8

56.3

1.8

13.1

38.4

16.7

31.8

$P<0.0001)$. To diminish risk of recall bias, we also present correlations using self-report post-restriction scores. As shown in Fig. 1(b) there is a positive correlation between post-restriction AUDIT scores such that as they increase so to do the post-restriction scores for: depression $(r=0.23, P<0.0001)$, anxiety $(r=0.19$, $P<0.001)$ and drinking to cope $(r=0.56, P<0.0001)$.

We used MLM to test how cohort characteristics (for example age, gender, concern about finances because of the pandemic) were associated with change in AUDIT scores and post-restriction

AUDIT scores. Each cohort factor was considered while accounting for the influence of all the other factors. Characteristics that significantly had an impact on both the change in AUDIT scores and post-restriction AUDIT scores are illustrated in Fig. 2. Both an increase in AUDIT score and higher post-restriction AUDIT score were associated with the age group 41-50 $(|t|=3.648, P<0.00$, $|t|=2.023, P<0.05$, respectively, where $t$ is the $t$ statistic of the multiple linear regression), participants that reported they had received a diagnosis of mental illness since the start of the COVID-19 restrictions $(|t|=3.163, P<0.01,|t|=3.963, P<0.0001$, respectively) and participants living in a residence with no garden $(|t|=3.163, P<0.01,|t|=2.096, P<0.05$, respectively).

Additionally, an increase in AUDIT score was associated with self-employed currently working individuals, furloughed individuals, or individuals with a confirmed COVID-19 diagnosis $(|t|=2.159$, $P<0.05,|t|=2.551, P<0.05,|t|=2.386, P<0.05$, respectively). However, occupation and COVID-19 diagnosis were not associated with post-restriction AUDIT score. Conversely, smoking status was associated with increase post-restriction AUDIT score $(|t|=4.467$, $P<0.0001)$ but not AUDIT change.

We undertook a one-way ANCOVA on data for the individuals whose AUDIT score had increased compared with those whose drinking had decreased with implementation of COVID-19 restrictions, while controlling for post- restriction AUDIT score to mitigate the effect of RTM (Fig. 3(a)). There was a significant effect of group (increased versus decreased) on pre-restriction AUDIT score $(F(2,332)=119, P<0.0001)$. However, of particular interest was the result of the post hoc tests that revealed that those in the group who increased their AUDIT score had a significantly lower average AUDIT score before the COVID-19 restrictions, than individuals who decreased their AUDIT score $(P<0.0001)$.

A two-way ANOVA was used to assess if 'coping' as a motivation to drink differed between those whose AUDIT score had increased compared with those in whom it decreased (Fig. 3(b)). As expected, we found a significant effect of group (increased versus decreased) $(F(1,536)=11.11, P<0.001)$ and restriction (pre- versus post-COVID-19) $(F(1,536)=4.21, P<0.05)$. The post hoc Tukey's multiple comparison test revealed no significant difference in coping scores before the COVID- 19 restrictions between individuals who had an increased versus a decreased AUDIT score $(P=0.99)$. However, as expected post-COVID-19 restriction coping score was significantly higher compared with pre-COVID-19 restriction coping score in those in the group who increased their AUDIT score $(P<0.01)$.

Finally, we looked at the characteristics of those whose AUDIT score had increased with those whose score had decreased (Supplementary Material 4). The ratio of males and females were similar in both groups (increased: female 62.8\%, male 35.9\% $v$. decreased: female $65.1 \%$, male $33.9 \%$ ). Interestingly, a higher proportion of individuals with a previous history of alcohol dependence were in the increased group (10.3\%) compared with the decreased group (3.6\%). Regarding age, AUDIT scores decreased largely in younger participants whereas all other ages were associated with an increased score.

\section{Discussion}

\section{Main findings}

We found that although COVID-19 restrictions have overall reduced AUDIT scores within our cohort, almost a quarter (23.2\%) reported an increase. We found that there were significant positive correlations between anxiety and depression scores and increasing AUDIT scores, consistent with recent evidence that found alcohol intake was associated with higher depression, 
Table 2 Measures pre- versus post-COVID-19 restrictions ${ }^{a}$

\begin{tabular}{|c|c|c|c|c|c|}
\hline & Pre-restriction, mean (s.d.) & Post-restriction, mean (s.d.) & $z$ & $P$ & Direction of change \\
\hline GAD-7 score & $4.5(4.7)$ & $6.3(5.5)$ & -8.2 & $<0.0001$ & Increased \\
\hline PHQ-9 score & $3.7(4.3))$ & $6.6(5.8)$ & -11.2 & $<0.0001$ & Increased \\
\hline AUDIT score & $8.6(5.3)$ & $6.7(5.6)$ & -7.8 & $<0.0001$ & Decreased \\
\hline Consumption frequency score & $2.8(0.8)$ & $2.7(1.12)$ & -1.9 & 0.053 & Decreased \\
\hline Number of drinks score & $0.9(1.0)$ & $0.7(0.9)$ & -3.7 & $<0.0001$ & Decreased \\
\hline Binge frequency score & $1.5(1.1)$ & $1.1(1.2)$ & -6.3 & $<0.0001$ & Decreased \\
\hline Hangover frequency & $2.7(1.8)$ & $2.4(2.2)$ & -3.6 & $<0.0001$ & Decreased \\
\hline Hangover sensitivity & $1.6(1.0)$ & $1.2(1.2)$ & -5.9 & $<0.0001$ & Decreased \\
\hline DMQ: social & $14.7(5.2)$ & $10.2(5.2)$ & -13.1 & $<0.0001$ & Decreased \\
\hline DMQ: coping & $9.0(3.8)$ & $9.2(4.6)$ & -0.2 & 0.875 & Increased \\
\hline DMQ: enhancement & $13.2(5.0)$ & $12.2(5.3)$ & -4.8 & $<0.0001$ & Decreased \\
\hline DMQ: conformity & $7.3(3.0)$ & $6.1(2.6)$ & -8.6 & $<0.0001$ & Decreased \\
\hline
\end{tabular}

anxiety and stress symptoms. ${ }^{21-23}$ The largest increase in consumption was seen in those who reported increased 'drinking to cope' as a motivation. Notably, this 'coping' motivation also correlated with both increased anxiety and depression scores because of COVID19 restrictions. With regards to alcohol consumption, participants did not report a change in how often they were consuming alcohol but did report a significant reduction in the number of drinks and a reduction in binge drinking (six or more drinks on one drinking occasion).

\section{Interpretation of our findings}

When comparing the pre-restriction AUDIT scores of those individuals who increased versus decreased their drinking, those individuals whose AUDIT score decreased, initially consumed more alcohol than those whose score increased. Those individuals who had an increased AUDIT score also had a significant increase in their motivation to drink to cope score compared with those that had a decreased AUDIT score. This is consistent with the selfmedication hypothesis ${ }^{24,25}$ and shows that COVID-19 restrictions have led to an increase in coping-motivated drinking, within these individuals. Of concern was that a higher proportion of participants with a self-reported history of alcohol dependence had an increased AUDIT score after the implementation of COVID-19 restrictions that likely reflects relapse. Future studies should evaluate if the pandemic is leading to higher relapse rates in recovering alcoholics.
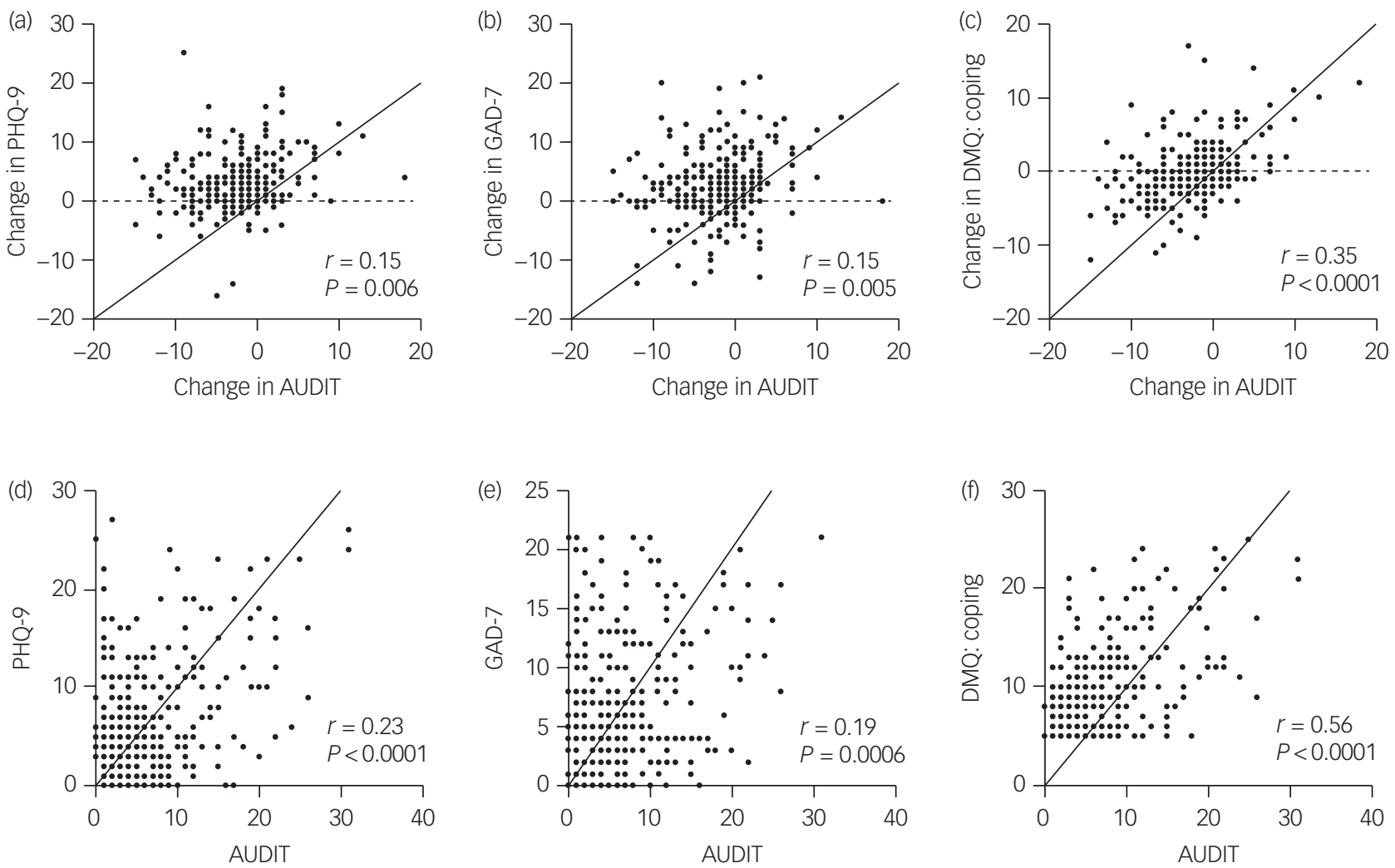

Fig. 1 Correlation of change in (a) anxiety, (b) depression and (c) motivation to drink to cope with change in scores of Alcohol Use Disorders Identification Test (AUDIT) given the impact on coronavirus disease 2019 (COVID-19) restrictions. Current (during the pandemic, post-COVID restriction implementation) self-report scores for (d) anxiety, (e) depression and (f) motivation to drink to cope correlated with current AUDIT scores.

Correlation analysis used Spearman's rank correlations. GAD, Generalized anxiety disorder; PHQ, Patient Health Questionnaire, DMQ, Drinking Motives Questionnaire. 


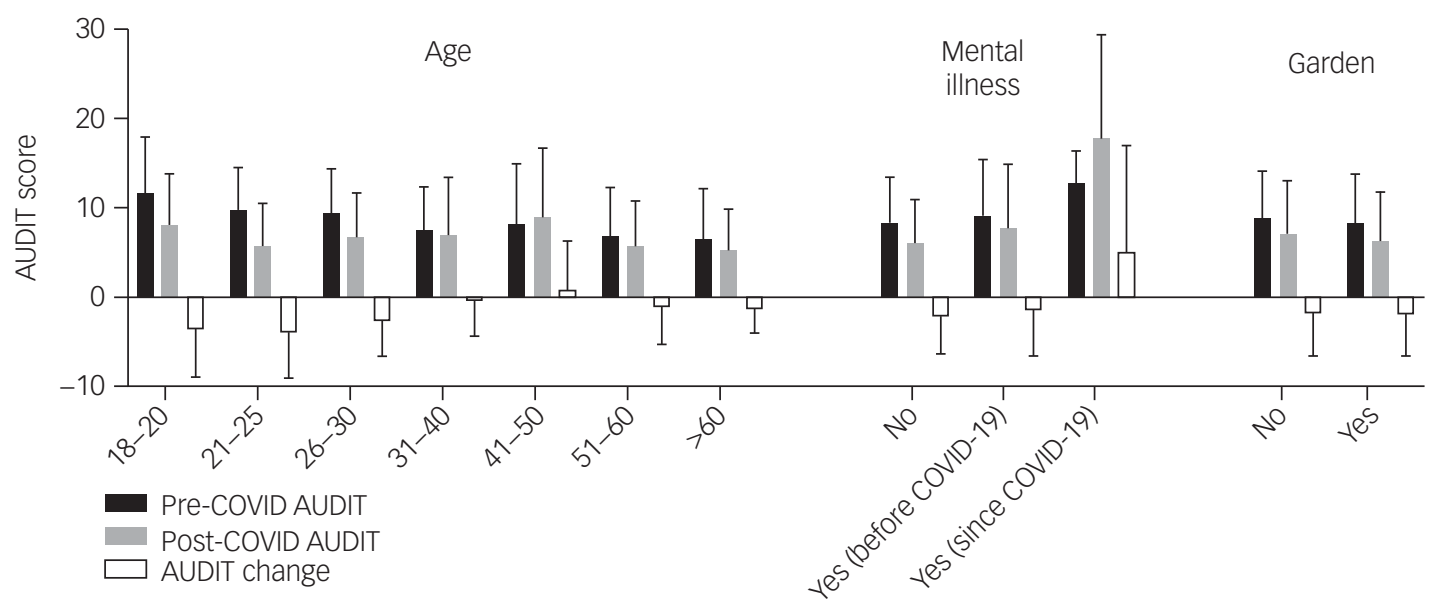

Fig. 2 Impact of cohort factors on pre- (black), post- (grey) and change (white) in scores of Alcohol Use Disorders Identification Test (AUDIT) since the implementation of coronavirus disease 2019 (COVID-19) restrictions.

Data collected from 336 survey participants. Data shown as mean (s.d.).

A range of other factors were associated with increased AUDIT scores including age, a diagnosis of mental illness since the onset of the pandemic, currently working self-employed or furloughed staff, a confirmed positive COVID-19 diagnosis, smoking status and living in a residence without a garden. Notably most participants whose AUDIT score decreased were in the younger age groups, likely because of reduced social activities. Indeed, we attribute the disparity between our pre-restriction self-report AUDIT scores and the population data from the 2014 APMS to our participants being largely from younger age groups.

We found that those currently working self-employed or furloughed staff was associated with increased consumption. This is unsurprising as these groups are the most likely to experience the largest financial/job- related concern. It has been previously shown that low job control and high demands at work are related to higher levels of drinking to cope. ${ }^{26}$ Additionally, studies have shown that workers who felt their skills were underused and/or were not involved in decision-making, as many furloughed staff must have experienced, had higher levels of heavy drinking. ${ }^{27}$ Furthermore, the association we found between a diagnosis of mental illness since the onset of the pandemic or a confirmed positive COVID-19 diagnosis with increased AUDIT score may be explained also by the self-medication hypothesis to alleviate anxiety and stress. ${ }^{28,29}$
Our respondents were predominantly female though we found no association between gender and AUDIT change or post-AUDIT score. This differs from other studies that found that COVID-19 psychological distress resulted in increased drinking only in females. ${ }^{29,30}$ This may be because of the younger average age of our cohort (58.5\% aged 21-30) compared with the average age of the previous study (41.7 years). Future research should evaluate the effect of age in drinking-related gender differences in response to psychological distress. Although we found that AUDIT scores overall were reduced in our cohort, scores did increase in a substantial minority. Other surveys have reported an increase in consumption of $20-30 \%$ in UK, Australia and Belgium as a result of COVID restrictions. ${ }^{5,6,21,30-32}$ However, our finding of an overall reduction in alcohol consumption is supported by an Australian study that reported the lowest levels of the alcohol metabolite ethyl sulphate in wastewater in April 2020 compared with previous years. ${ }^{33}$

\section{Limitations}

The cross-sectional self-report nature of the study is a major limitation as it is subject to reporting, self-perception and recall bias. We attempted to mitigate recall bias by analysing both perceived change in outcome scores and post-restriction scores (as current behaviour should be less effected by recall bias). We are also cautious about (a)

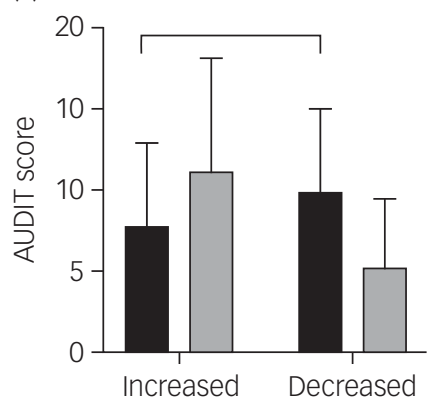

(b)

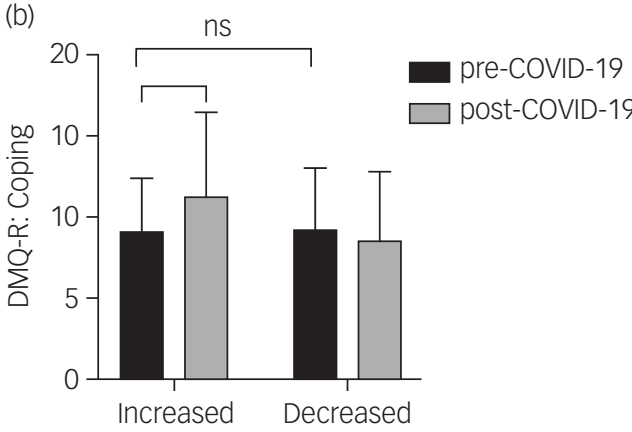

Fig. 3 Comparison of Alcohol Use Disorders Identification Test (AUDIT) (a) and drinking to cope (b) scores between individuals who increased or decreased alcohol consumption since implementation of coronavirus disease 2019 (COVID-19) restrictions.

For each group (increased versus decreased) self-report scores are shown pre-COVID-19 (black) and post-COVID-19 (grey) restrictions. Data presented is mean (s.d.) of 336 survey participants. *Significant at $P<0.05 ;$ ns, non-significance. 
inferring that the increased self-report scores relate to increases in clinically significant levels of anxiety and depression. Further, we acknowledge that the AUDIT is designed as a screening instrument for recent alcohol-related behaviours rather than to assess previous behaviours, however, we felt it was the best instrument to use to compare our study with other studies.

We acknowledge our relatively small sample size and our online sampling strategy are key limitations of this study. Additionally, the lack of ethnic diversity and the high education level of respondents means that findings are not necessarily generalisable to the general population. Given the increased risk COVID-19 presents to those from Black and ethnic minority backgrounds, ${ }^{34}$ future studies should assess changes in alcohol consumption in these individuals. Future studies should aim to assess how COVID-19 restrictions altered drinking behaviours in a larger and more representative sample. Additionally, caution should be taken when interpreting comparisons with the AMPS data-set as the AMPS only included UK residents whereas our study did not. Similarly, the AMPS had a much larger more diverse cohort and employed more stringent recruitment than the current study.

\section{Implications}

Restrictions to reduce the spread of COVID-19 significantly reduced overall AUDIT scores. This reduction was driven by a decrease in the quantity of alcohol consumption, whereas the frequency remained unchanged. Our data suggests this is likely the result of a reduction in social and societal norms that encourage drinking behaviour as we saw a reduction in motivation scores for the categories: social, enhancement and conformity. Our data showing that increased AUDIT scores are associated with increased anxiety, depression and 'drinking to cope' provides support to the prediction that pandemic-induced distress would increase alcohol consumption in some individuals whereas for others a reduction resulted from a decrease in physical and financial availability of alcohol.

Alcohol consumption increased in those respondents who reported increasing anxiety and depression levels and, most importantly, 'drinking to cope' with COVID-19 restrictions. This increase is of immense public health concern, given the consequences of problem drinking. Notably, the COLLATE study in Australia used similar assessment methods to identify individuals most likely to require support because of their increased drinking. ${ }^{35}$ We also found that individuals in the age range 41-50, a diagnosis of mental illness since the onset of the pandemic, currently working self-employed or furloughed staff, a confirmed positive COVID19 diagnosis, smoking status and living in a residence without a garden were associated with increased AUDIT scores post-restrictions. Changes in alcohol consumption behaviours require further analysis as the pandemic progresses in order to evaluate any longterm consequences of this behavioural shift and further restrictions. Further understanding of alcohol consumption in these groups would help target prevention and treatment initiatives.

\footnotetext{
Emily O. C. Palmer D, Division of Psychiatry, Department of Brain Science, Imperial College London, UK; William Trender, The Computational, Cognitive and Clinical Neuroimaging Laboratory, Imperial College London, UK; Robin J. Tyacke, Division of Psychiatry, Department of Brain Science, Imperial College London, UK; Adam Hampshire, The Computational, Cognitive and Clinical Neuroimaging Laboratory, Imperial College London, UK; Anne Lingford-Hughes, Division of Psychiatry, Department of Brain Science, Imperial College London, UK

Correspondence: Emily Palmer. Email: e.palmer@imperial.ac.uk

First received 18 Mar 2021, final revision 15 Jul 2021, accepted 1 Aug 2021
}

\section{Supplementary material}

Supplementary material is available online at https://doi.org/10.1192/bjo.2021.986

\section{Data availability}

The data are available from the corresponding author upon request.

\section{Author contributions}

E.O.C.P., R.J.T., A.L.-H. and A.H. contributed to the conceptualisation and design of the surveys. W.T. created the script for the survey and the host website. E.O.C.P coordinated the writing and editing of the manuscript and analysed the data. All authors approved the final version for submission.

\section{Funding}

This research was supported by an unrestricted donation from Alcarelle Ltd to Imperial College London.

\section{Declaration of interest}

R.J.T. works as a consultant for Alcarelle Ltd. This research was conducted as part of a postgraduate course funded by an unrestricted grant from Alcarelle Ltd to Imperial college London, this is supervised by A.L.H. A.L.-H. has received funds to support research from Lundbeck and GSK, and for chairing/presenting Lundbeck. There are no other conflicts of interest.

\section{References}

1 Office of National Statistics. Coronavirus and Anxiety, Great Britain: 3 April 2020 to 10 May 2020. ONS, 2020.

2 O'Connor RC, Wetherall K, Cleare S, McClelland H, Melson AJ, Niedzwiedz CL, et al. Mental health and wellbeing during the COVID-19 pandemic: Iongitudinal analyses of adults in the UK COVID-19 Mental Health \& Wellbeing study. $\mathrm{Br} J$ Psychiatry [Epub ahead of print] 21 Oct 2020. https://doi.org/10.1192/bjp. 2020.212.

3 Holmes EA, O'Connor RC, Perry VH, Tracey I, Wessely S, Arseneault L, et al. Multidisciplinary research priorities for the COVID-19 pandemic: a call for action for mental health science. Lancet Psychiatry 2020; 6: 547-60.

4 Calvey T, Scheibein F, Saad NA, Shirasaka T, Dannatt L, Stowe MJ, et al. The changing landscape of alcohol use and alcohol use disorder during the COVID-19 pandemic - perspectives of early career professionals in 16 Countries. J Addict Med 2020; 14: e284-e286.

5 Vanderbruggen N, Matthys F, Van Laere S, Zeeuws D, Santermans L, Van den Ameele $S$, et al. Self-reported alcohol, tobacco, and cannabis use during COVID-19 lockdown measures: results from a web-based survey. Eur Addict Res 2020; 26: 309-15.

6 Royal College of Psychiatrists. Next Steps for Funding Mental Healthcare in England Prevention. Royal College of Psychiatrists, 2020.

7 Rubin R. Alcohol-related diseases increased as some people drank more during the COVID-19 pandemic. JAMA 2021; 326: 209-11.

8 Sharma A, Kroumpouzos G, Lotti T, Goldust M. COVID-19 and alcohol use. Drug Alcohol Rev 2021; 40: 683-4.

9 Office for National Statistics. Quarterly Alcohol-Specific Deaths in England and Wales: 2001 to 2019 Registrations and Quarter 1 (Jan to Mar) to Quarter 4 (Oct to Dec) 2020 Provisional Registrations. ONS, 2021.

10 Beck KH, Thombs DL, Mahoney CA, Fingar KM. Social context and sensation seeking: gender differences in college student drinking motivations. Int $J$ Addict 1995; 30: 1101-15.

11 Prescott CA, Cross RJ, Kuhn JW, Horn JL, Kendler KS. Is risk for alcoholism mediated by individual differences in drinking motivations? Alcohol Clin Exp Res 2004; 28: 29-39.

12 Paton A, Fooks G, Maestri G, Lowe P. Submission of Evidence on the Disproportionate Impact of COVID 19, and the UK Government Response, on Ethnic Minorities and Women in the UK. Aston University, 2020.

13 Saunders JB, Aasland OG, Babor TF, De la Fuente JR, Grant M. Development of the alcohol use disorders identification test (AUDIT): WHO collaborative project on early detection of persons with harmful alcohol consumption-II. Addiction 1993; 88: 791-804.

14 Public Health England. Guidance Alcohol Use Screening Tests. Public Health England,2017 (https://www.gov.uk/government/publications/alcohol-use-screening-tests).

15 Kroenke K, Spitzer RL, Williams JB. The PHQ-9: validity of a brief depression severity measure. J Gen Intern Med 2001; 16: 606-13. 
16 Spitzer RL, Kroenke K, Williams JB, Löwe B. A brief measure for assessing generalized anxiety disorder: the GAD-7. Arch Intern Med 2006; 166: 1092-7.

17 Plummer F, Manea L, Trepel D, McMillan D. Screening for anxiety disorders with the GAD-7 and GAD-2: a systematic review and diagnostic metaanalysis. Gen Hosp Psychiatry 2016; 39: 24-31.

18 Cooper ML. Motivations for alcohol use among adolescents: development and validation of a four-factor model. Psychol Assess 1994; 6: 117.

19 McManus S, Bebbington P, Jenkins R, Brugha T. Adult Psychiatric Morbidity Survey: Survey of Mental Health and Wellbeing, England, 2014. NHS Digital, 2014.

20 McManus S, Bebbington $\mathrm{P}$, Jenkins R, Brugha T. Adult Psychiatric Morbidity Survey: Survey of Mental Health and Wellbeing, England, 2014. NHS Digital, 2016.

21 Stanton R, To QG, Khalesi S, Williams SL, Alley SJ, Thwaite TL, et al. Depression, anxiety and stress during COVID-19: associations with changes in physical activity, sleep, tobacco and alcohol use in Australian adults. Int J Environ Res Public Health 2020; 17: 4065.

22 Lechner WV, Laurene KR, Patel S, Anderson M, Grega C, Kenne DR. Changes in alcohol use as a function of psychological distress and social support following COVID-19 related University closings. Addict Behav 2020; 110: 106527.

23 Wardell JD, Kempe T, Rapinda KK, Single A, Bilevicius E, Frohlich JR, et al. Drinking to cope during COVID-19 pandemic: the role of external and internal factors in coping motive pathways to alcohol use, solitary drinking, and alcohol problems. Alcohol Clin Exp Res 2020; 44: 2073-83.

24 Khantzian EJ. The self-medication hypothesis of substance use disorders: a reconsideration and recent applications. Harv Rev Psychiatry 1997; 4: 231-44.

25 Cooper ML, Kuntsche E, Levitt A, Barber LL, Wolf S. Motivational models of substance use: a review of theory and research on motives for using alcohol, marijuana, and tobacco. 2016. The Oxford handbook of substance use and substance use disorders. (pp. 375-421). Oxford University Press.

26 Martin JK, Roman PM, Blum TC. Job stress, drinking networks, and social support at work: a comprehensive model of employees' problem drinking behaviors. Sociol Q 1996; 37: 579-99.
27 Greenberg ES, Grunberg L. Work alienation and problem alcohol behavior. J Health Soc Behav 1995; 36: 83-102.

28 Anthenelli R, Grandison L. Effects of stress on alcohol consumption. Alcohol Res 2012; 34: 381-2.

29 Rodriguez LM, Litt DM, Stewart SH. Drinking to cope with the pandemic: the unique associations of COVID-19-related perceived threat and psychological distress to drinking behaviors in American men and women. Addict Behav 2020: 110: 106532

30 Garnett C, Jackson S, Oldham M, Brown J, Steptoe A, Fancourt D. Factors associated with drinking behaviour during COVID-19 social distancing and lockdown among adults in the UK. Drug Alcohol Depend 2021; 219: 108461.

31 Alcohol Change UK. Drinking during Lockdown: Headline Findings. Alcohol Change UK, 2020 (https://alcoholchange.org.uk/blog/2020/covid19-drinkingduring-lockdown-headline-findings).

32 Jacob L, Smith L, Armstrong NC, Yakkundi A, Barnett Y, Butler L, et al. Alcohol use and mental health during COVID-19 lockdown: a cross-sectional study in a sample of UK adults. Drug Alcohol Depend 2021; 219: 108488.

33 Bade R, Simpson BS, Ghetia M, Nguyen L, White JM, Gerber C. Changes in alcohol consumption associated with social distancing and self-isolation policies triggered by COVID-19 in South Australia: a wastewater analysis study. Addiction 2020; 116: 1600-1605

34 Trivedy C, Mills I, Dhanoya O. The impact of the risk of COVID-19 on Black, Asian and Minority Ethnic (BAME) members of the UK dental profession. Br Dent $J$ 2020; 228: 919-22

35 Neill E, Meyer D, Toh WL, van Rheenen TE, Phillipou A, Tan EJ, et al. Alcohol use in Australia during the early days of the COVID-19 pandemic: initial results from the COLLATE project. Psychiatry Clin Neurosci 2020; 74: 542-549.

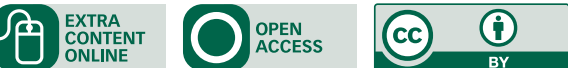

